\title{
Novel Engineered Human Fluorescent Osteoblasts for Scaffolds Bioassays
}

\author{
Katia Campioni ${ }^{1}$, Cristina Morelli ${ }^{1}$, Antonio D'Agostino ${ }^{2}$, Lorenzo Trevisiol ${ }^{2}$, Pier Francesco Nocini ${ }^{2}$, \\ Marco Manfrini ${ }^{1}$, Mauro Tognon ${ }^{1^{*}}$
}

${ }^{1}$ Section of Cell Biology and Molecular Genetics, School of Medicine and Centre of Biotechnology, University of Ferrara, Ferrara, Italy; ${ }^{2}$ Department of Odontostomatology and Maxillo-Facial Surgery, School of Medicine and School of Dentistry, University of Verona, Verona, Italy.

Email: *tgm@unife.it

Received July $7^{\text {th }}, 2010$; revised July $28^{\text {th }}, 2010$; accepted July $29^{\text {th }} 2010$.

\begin{abstract}
Many cellular models have been used to investigate bone substitutes including coral-derived hydroxylapatite (HA). The aim of this study was to verify whether a new cellular model represented by Saos-eGFP cells can be used to test biomaterials by in vitro assays. Saos-eGFP cells which express the enhanced Green Fluorescent Protein (eGFP), were derived from the osteoblast-like cell line Saos-2. To this purpose Saos-eGFP cells were employed to investigate the in-vitro bioactivity of a well characterized coral-derived HA biomaterial, in block and granule forms. This engineered cell line, by evaluating the emitted fluorescence, allowed us to assay 1) cell adhesion, 2) cell proliferation and 3) colony capability. Electron microscopy analysis was employed to evaluate the 4) morphology of cells seeded on the biomaterial surface. 5) Histological analysis of the bone grown after scaffold implantation was carried out in specimens from two clinical cases. Saos-eGFP cells indicate, as established before, that the coralline-HA biomaterial has a good in vitro cytocompatibility when tested with human osteoblast-like cells. Some differences in proliferation activity were detected for the two different forms assayed. Cytocompatibility data from in vitro analyses were confirmed by in vivo behaviour of biomaterials. Our tests suggest that the engineered cell line Saos-eGFP represents a suitable in vitro mode for studying the biocompatibility, the cell adhesion, spreading and proliferation on biomaterials developed for clinical applications. The main advantages of this cellular model are 1) less time consuming and 2) a reduced cost of the experiments.
\end{abstract}

Keywords: Fluorescence, Osteoblast, Biomaterial

\section{Introduction}

Investigations into bone substitutes have been addressed to different biomaterials including coral-derived hydroxylapatite (HA). One of the main goals of this biomaterial, which is similar in its chemical composition to human bone, is to modulate cellular responses which control the interaction with the scaffold. Specifically, it may induce spontaneous three dimensional self-organisation in the new tissue, as it has been observed in the physiological environment [1,2]. Indeed, cell behaviour and phenotype are governed by responses to different types of signals that include mechanical forces, electrical stimuli, and various physical cues [3]. In addition, cells sense and respond to a variety of signals including soluble growth factors, differentiation factors, cytokines, and ion gradients [4-6]. Materials employed as scaffolds must possess specific features, such as cyto- and bio-compatibility, osteo-inductivity and -conductivity, as well as the right mechanical strength to provide structural support during tissue growth and remodelling. Besides those of a bovine origin, natural HA biomaterials can also be derived from coral exoskeletons (genus Porites and Goniopora). Hydrothermal treatment $\left(260^{\circ} \mathrm{C}\right.$.; $\left.15,000 \mathrm{PSI}\right)$ of the calcium carbonate exoskeletal microstructure of these corals results in conversion into hydroxylapatite [7]. Different hydrothermal coral treatments have resulted in only partial conversion of its calcium carbonate to HA [8]. As a result, its $\mathrm{HA} / \mathrm{CaCO}_{3}$ composite is resorbed faster than pure HA. Like natural bone, this HA may contain minor elements such as $\mathrm{Mg}, \mathrm{Sr}, \mathrm{F}$, and $\mathrm{CO}_{3}$ and has a completely interconnected porosity which is similar to trabecular bone. 
Coral-derived HA biomaterial in block and granule forms, as well as other types of HA materials, have been well characterized for many parameters including their cytocompatibility using different cellular models [9-11]. These cellular models however are time-consuming, with high costs and tedious to be used.

The aim of this study was to investigate whether a new cellular model known as Saos-eGFP, derived from the osteoblast-like cell line Saos-2, can be employed for the in-vitro bioactivity of coral-derived HA biomaterial in block and granule forms. Saos-eGFP is a genetically engineered cell line obtained from Saos-2 osteosarcoma cells which express the enhanced Green Fluorescent Protein (eGFP) [12,13]. Saos-eGFP cellular model allowed us to assay 1) cell adhesion, 2) cell proliferation and 3) colony capability, by evaluating their emitted fluorescence. We also evaluated the 4) morphology of the cells seeded on the biomaterial surface by employing electron microscopy. In addition, 5) histological analyses of bone grown in vivo after scaffold implantation are presented.

Our data indicate that human Saos-eGFP fluorescent cells employed to test the well characterized coral HA, as a standardized biomaterial, is a good cellular model to characterize scaffolds.

\section{Materials and Methods}

\subsection{Saos-eGFP Cell line}

An engineered human osteoblast-like cell line, known as Saos-eGFP, was obtained from parental Saos-2 cells as described elsewhere $[12,13]$. In brief, Saos-eGFP cells were cultured in DMEM-F12 (BioWhittaker, Milan, Italy) supplemented with $10 \%$ fetal bovine serum (BioWhittaker, Milan, Italy), $500 \mu / \mathrm{ml}$ penicillin/streptomycin (Sigma, Milan, Italy), and maintained in a humidified atmosphere at $37^{\circ} \mathrm{C}$ containing $5 \% \mathrm{CO}_{2}$. The antibiotic Genetycin (500 ug/ml), known as G418 (Invitrogen, Milan, Italy) was employed to keep in selection Saos-2 cells, engineered with the recombinant plasmid vector expressing the enhanced green fluorescent protein (eGFP) which carries the resistance gene (Neo) to the antibiotic (Figure 1). A known number of Saos2-eGFP $\left(5 \times 10^{3}, 10\right.$ $\left.\times 10^{4}, 20 \times 10^{4}, 40 \times 10^{4}, 80 \times 10^{4}, 16 \times 10^{5}\right)$ was seeded and cultured for 24 hours in 24-well plates $(\varnothing=10 \mathrm{~mm})$. A calibration curve was obtained by reporting the number of cells present in each sample, which emitted fluorescence, on a graph (excitation $\lambda=488 \mathrm{~nm}$, emission $\lambda$ $=508 \mathrm{~nm}$ ) (Figure 2).

\subsection{Biomaterial}

The assayed biomaterial, which is commercially available, is made with marine coral exoskeleton material,

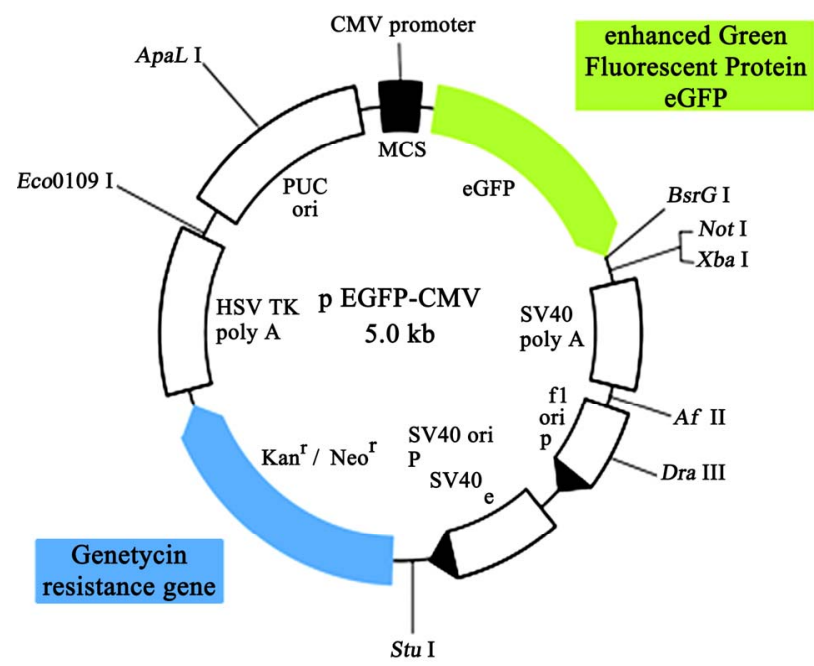

Figure 1. pEGFP plasmid vector showing the eGFP insertion site and the Neo resistance gene.

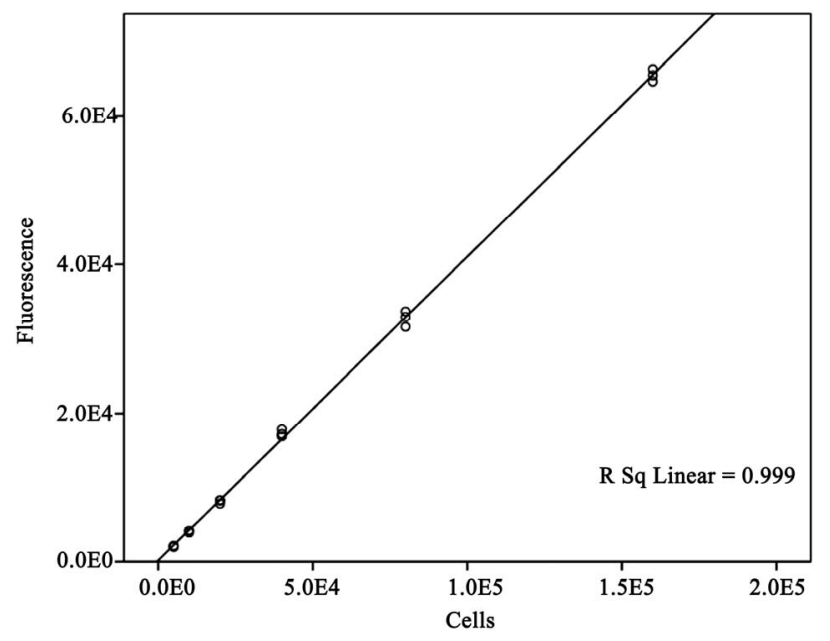

Figure 2. The calibration curve was obtained by seeding Saos-eGFP cells at different densities and measuring the fluorescence emitted (excitation $\lambda=488 \mathrm{~nm}$, emission $\lambda=$ $508 \mathrm{~nm}$ ). $R$ value showed a good positive correlation between cells number and fluorescence emitted.

hydrothermally converted to hydroxylapatite (HA). HA scaffolds are indicated for use as cancellous bone substitutes or as augmentation material for repairing bone defects, in combination with autologous bone, allografts, blood or bone marrow. This HA material is routinely used in oral-maxillo-facial surgery clinical practice for bone regeneration. In our cyto-compatibility assays the biomaterial was employed both in small blocks $(10 \times 10$ $\times 10 \mathrm{~mm})$ and granules $(1 \div 4 \mathrm{~mm})$.

\subsection{Scaffold Cell Loading}

Proliferating Saos-eGFP cell monolayers, at $50 \div 60 \%$ 
confluence, were detached from the culture flasks and re-suspended in culture medium to obtain a cell suspension with a density of $10^{4}$ cells $/ \mathrm{cm}^{2}$ surface area, in $1 \mathrm{ml}$, for each scaffold. A tissue culture polystyrene (TCPS) vessel was employed as control. The scaffold was then placed in wells $(\varnothing=10 \mathrm{~mm})$ filled with the proper cell suspension and incubated for $2 \mathrm{~h}$. To maximize cellscaffold interaction, cell suspension was subjected to pipetting every $15 \mathrm{~min}$. After the incubation period, scaffolds were placed in empty wells. Fresh culture medium, $1 \mathrm{ml}$, was added to each scaffold.

\subsection{Cell Adhesion and Proliferation}

Saos-eGFP cells were loaded onto scaffolds and TCPS (control), in 24-well culture plates $(\varnothing=10 \mathrm{~mm})$ and cultured as described above. In order to mimic the clinical application environment, the same volume of bone void filler was used for each type of scaffold. To analyze cell adhesion on each biomaterial and on the control, samples were incubated for $24 \mathrm{~h}$ at $37^{\circ} \mathrm{C}, 5 \% \mathrm{CO}_{2}$. Attached cells per scaffolds were detected by measuring the fluorescence (excitation $\lambda=488 \mathrm{~nm}$, emission $\lambda=508 \mathrm{~nm}$ ) emitted by the viable cells. Then, cells were re-fed with fresh culture medium and cultured at $37^{\circ} \mathrm{C}$ in a humidified atmosphere with $5 \% \mathrm{CO}_{2}$. Assays were carried out to evaluate cells attached on biomaterials and on the control, at $24 \mathrm{~h}, 48 \mathrm{~h}$, and $96 \mathrm{~h}$.

\subsection{Cell Spreading}

Saos-eGFP cells were loaded onto the scaffolds and TCPS, in 24-well culture plates $(\varnothing=10 \mathrm{~mm})$ and cultured as described above. Samples were incubated for 24 $\mathrm{h}, 48 \mathrm{~h}$ ad $96 \mathrm{~h}$ at $37^{\circ} \mathrm{C}, 5 \% \mathrm{CO}_{2}$. Direct observation of the living cells on the biomaterial was carried out by fluorescence microscopy in order to determine their distribution, colonization and morphology on the biomaterials.

\subsection{Scanning Electron Microscopy (SEM)}

For the SEM analysis, Saos-eGFP $\left(10^{4}\right.$ cells/well $)$ were cultured on HA scaffolds for $48 \mathrm{~h}$. Cells which were attached to the biomaterials were washed with PBS $1 \mathrm{X}$ solution and fixed for $1 \mathrm{~h}$ by $2.5 \%$ glutaraldehyde in a phosphate buffer and then for $4 \mathrm{~h}$ with a $1 \%$ osmium solution in a phosphate buffer. Specimens were coated with colloidal gold and analyzed using scanning electron microscopy (SEM, Cambridge UK, model Stereoscan S$360)$.

\subsection{Histology}

Granules were used for maxillary sinus augmentation in patients with bone resorption in the posterior segment of the upper jaw for implant-prosthetic rehabilitation.
Blocks were used as interpositional materials in orthognathic surgery patients when the upper jaw was down grafted in order to increase the stability of the mobilized segment. Biopsies were taken in accordance with the local ethical committee and after patients' written consent. In one clinical case (patient 1), biomaterial granules were used for bone regeneration in a patient who had undergone maxillary sinus augmentation for pre-prosthetic surgical rehabilitation. In this patient, a bone biopsy was taken four months after the first surgical procedure, during implant surgery. In another clinical case, (patient 2), to correct dentoskeletal deformities, blocks of biomaterial were employed as interpositional grafts in a patient treated with LeFort I osteotomy. Bone biopsies were taken from this patient, during plate removal, one year after surgery. Tissues were fixed in $4 \%$ formaldehyde and dehydrated in a graded series of alcohols. Then, all specimens were embedded in methylmethacrylate resin. Undecalcified sections were obtained using a water-cooled diamond saw. Slides were ground to a final thickness of about five $\mu \mathrm{m}$, placed on glass slides, stained with hematoxilin- eosin and viewed and photographed in a Leitz Orthoplan photomicroscope.

\subsection{Statistical Analysis}

All data were obtained from five independent experiments and expressed as a mean value $\pm \mathrm{SD}$. ANOVA testing followed by post-hoc Bonferroni testing was employed to evaluate differences in the mean values among groups. Statistically significant findings were considered when $\mathrm{p}<0.05$. All data elaboration was computed by SPSS (SPSS Inc., Chicago, IL, U.S.A.).

\section{Results}

\subsection{Cell Adhesion and Proliferation}

The results obtained showed a good adhesive capability of Saos-eGFP cells to the biomaterial. Indeed, SaoseGFP cells attached both to block and granules of the biomaterial with a prevalence of $67 \%$ and $64 \%$, respectively, compared to seeded cells. In the control experiment, $94 \%$ of the seeded cells were attached to the plastic well, showing a statistically significant difference $(\mathrm{p}<$ 0.01 ) as regards the two scaffolds (Figure 3). Cells proliferated in each sample during the assay as in the control $(p<0.01)$. At $48 \mathrm{~h}$, the fluorescence detected for the granules was lower than the fluorescence detected for the control and block $(p<0.01)$. Cells grown on blocks behaved differently, as shown by the data obtained at 96 incubation $\mathrm{h}$. Indeed at this point, the fluorescence detected on these samples diminished with statistically significant differences as regards the control and granules $(\mathrm{p}$ $<0.05)$ (Figure 4). 


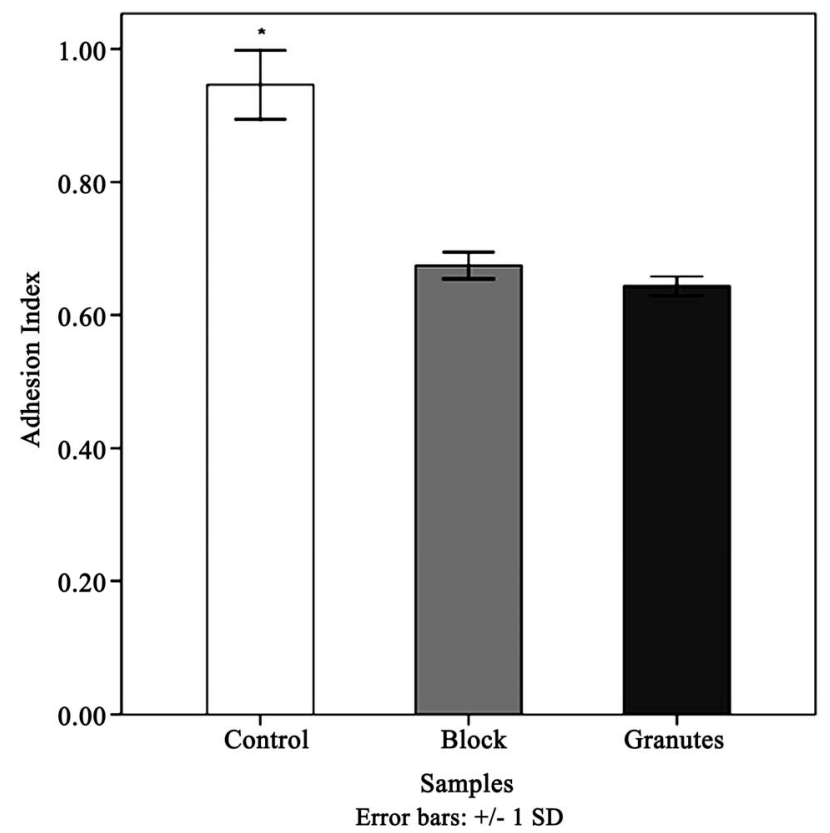

Figure 3. Adhesion index at 24 hours of incubation for controls and biomaterials, expressed as a ratio of living cells detected by fluorescence intensity measurement at 24 incubation hours, and cells seeded.

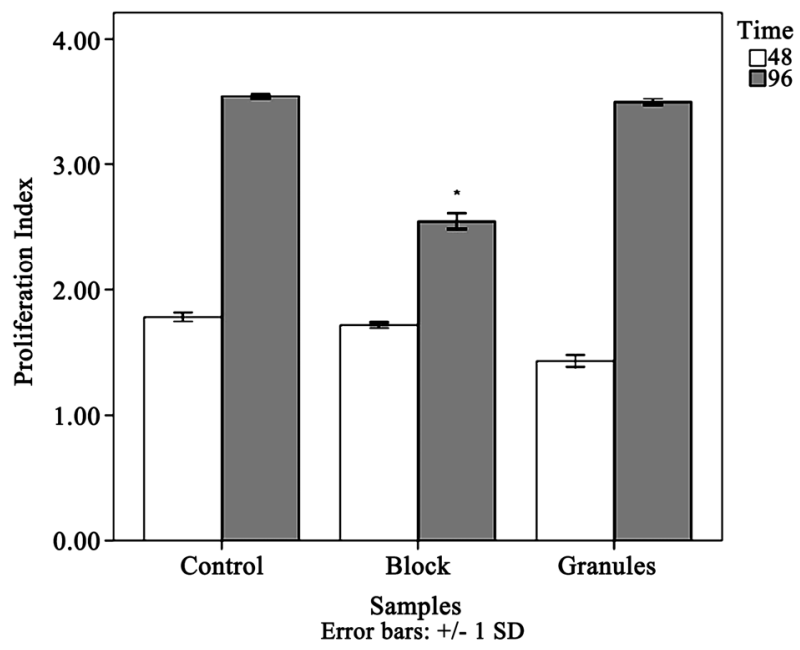

Figure 4. Proliferation index for control and biomaterials, expressed as a ratio of living cells detected by fluorescence intensity measured at 48 hours and 96 incubation hours respectively, and living cells detected at 24 incubation hours.

\subsection{Cell Spreading}

In the control experiment, the cell population was homogeneously distributed and appeared to have a normal morphology. Cells behaved similarly for both forms of the biomaterial. Indeed, the cells were able to colonize homogeneously on both granules and blocks (Figure 5).

\subsection{Scanning Electron Microscopy}

The results obtained with the SEM analysis indicate no difference in the morphology of the cells grown on the biomaterial under analysis, both granules and blocks, when compared to the control. SEM images revealed the presence of several cells anchored to the surface of the biomaterial by cytoplasmic bridges, which were similar to pseudopodia (Figure 6). In addition, a large amount of biomaterial debris was detected on the surface of the cell membrane. This debris was not present on the cells grown on glass, where the cell surface appeared homogeneous.

\subsection{Histological Evaluation}

Histological analysis (patient 1) showed new bone formation with resorption of the biomaterial. Indeed, fibroosseous tissue represented about $50 \%$ of the biopsy volume, while lamellar bone was $10 \%$ of the biopsy. Bone fatty marrow and vessels represented $40 \%$ of the biopsy. There was no evidence of inflammation or foreign body reactions. The histological evaluation (patient 2) showed,

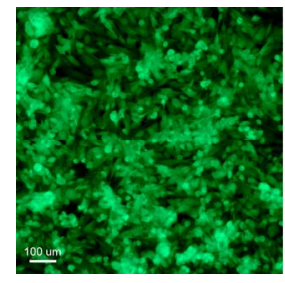

(a)

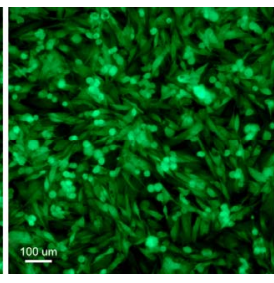

(b)

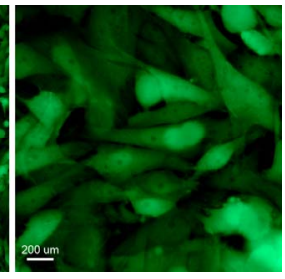

(c)
Figure 5. Direct observation by fluorescence microscope. At $48 \mathrm{~h}$ the Saos-eGFP cells appeared well attached to the control (a) as well as to the biomaterials. The assay revealed a homogeneous distribution of Saos-eGFP cells on the surfaces of the biomaterials, both the blocks (b) and granules (c).

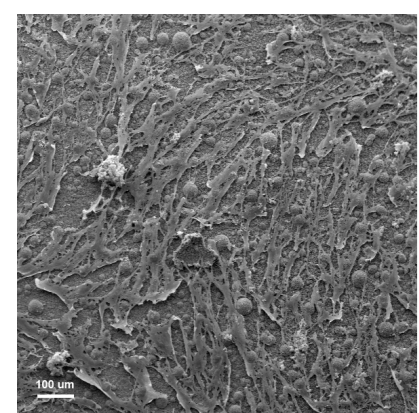

(a)

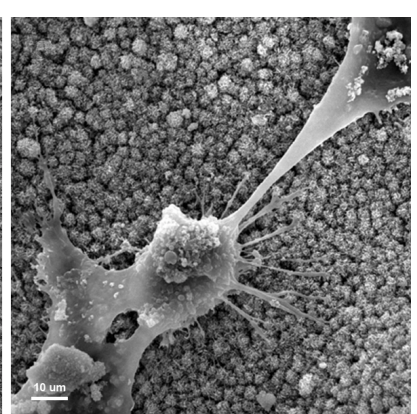

(b)
Figure 6. SEM images showed that at $48 \mathrm{~h}$, Saos-eGFP cells were homogeneously distributed on block biomaterials surface (a) and appear anchored to the granular scaffold by cytoplasmic bridges also involved in cells interaction (b). Debris is also evident on the cell surface. 
as expected, the residual biomaterial surrounded by mature compact bone. There was evidence of bone regrowth inside the biomaterial giving continuity between osteotomy sites with significant stability for the down grafted maxilla. This biomaterial represented $20 \%$ of the biopsy volume, while bone density was in the order of $40 \%$. The remaining $40 \%$ of the biopsy was represented by fibroosseous tissue. In this patient, a minor inflammation process with the presence of macrophages was revealed by histological analysis (Figure 7).

\section{Discussion}

Saos-eGFP cells were employed to determine mature osteoblastic cell morphology, spread and proliferation on coralline-HA biomaterials. Saos-eGFP on the biomaterial showed homogeneous spreading and a different rate of proliferation. This result demonstrates that composition, surface shape and biomaterial morphology, may influence the relationship between scaffold and cell. Indeed, Saos-eGFP cells which attached to the biomaterial, in the two forms assayed, showed a good adhesion ratio, although lower than for the control (TCPS). Biomaterial blocks appear to provide a better environment for adhesion compared to biomaterial granules, as shown by the percentage of adhered cells and by proliferation at 48 culture hours. Otherwise, the proliferation index determined for the biomaterial in blocks was lower than for granules at 96 incubation hours. Results showed that the granular form of the biomaterial has a greater capability to achieve Saos-eGFP cell proliferation. Direct observation at the fluorescence microscope showed that in the

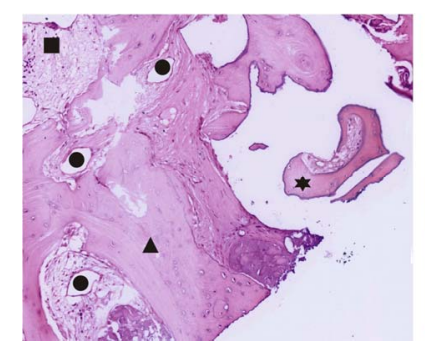

(a)

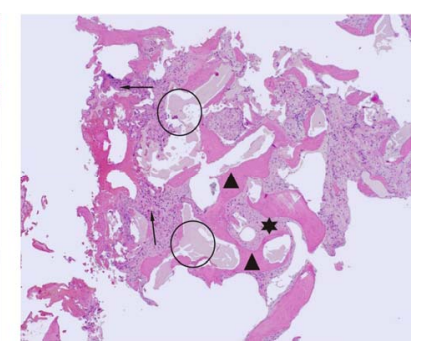

(b)
Figure 7. Histological evaluations indicate that tested biomaterial induces new bone formation, thus showing osteoconductive and osteoinductive properties. Patient 1 (a). Biopsy consist of cortical bone $(\Delta)$ associated with new bone formation and fibro-osseous tissue ( $\star$ ). Areas of cortical $(\Delta)$ and spongious bone $(\square)$ are both index of bone ingrowth together with neoangiogenetic processes demonstrated by vascular channels $(\Theta)$. Magnification 10x. Patient 2 (b). Black circles show exogenous material surrounded by mature cortical bone $(\Delta)$ and fibro - osseous $(\star)$ tissue associated with macrophages and the infiltration of inflammatory cells (black arrows). Magnification 40x. control experiment, the cell population was homogeneously distributed and appeared to have a normal morphology. Cells behaved similarly on both forms of the biomaterial until 96 cultivation hours. Indeed, cells were able to colonize homogeneously both granules and blocks forming colonies on the surface and on the trabecular structure of the biomaterials. SEM images revealed the presence of several cells anchored to the surface of the biomaterial by cytoplasmic bridges, which appeared similar to pseudopodia, confirming the capability of the biomaterial to achieve cell adhesion. The presence of debris, absent on cells grown on glass, observed at 48 and incubation hours, is probably an additional cause for the diminished number of cells grown on the block after that incubation period.

Histological evaluations indicated that both biomaterial granules and blocks showed osteoconductive properties, inducing new bone formation. From a clinical point of view, the osteoconductive properties were confirmed by the stability of the endosseous implants inserted in the posterior segment of the maxilla and in down grafted maxilla.

The possibility of learning which biomaterial leaves cell morphology, spread and proliferation unaltered, would be an important step towards obtaining bone tissue regeneration by employing three dimensional scaffolds. This knowledge is fundamental to oral and maxillo-facial surgery, which uses different types of biomaterials, in particular for treating specific pathologies, such as jaw bone atrophies, periodontal defects and cystic lesions.

In this study, we have described the use of Saos-eGFP engineered cell lines employed for the preliminary in vitro characterization of cell morphology, spread and proliferation on biomaterials, and we have clinically demonstrated bone re-growth driven by the biomaterials tested herein. The coralline-HA biomaterials, tested with Saos-eGFP cells, showed good cyto-compatibility although some differences in proliferation activity was found as reported earlier [14]. This cellular model will allow in vivo testing only for those materials which have shown the minimal required in vitro properties for successful bone tissue regeneration: an absence of cyto-toxic effects and good adhesion, spread and proliferation capabilities. This study has demonstrated that human engineered osteoblast-like cells expressing the eGFP is a suitable cellular model to assay biomaterials. Saos-eGFP has the advantage to test biomaterials by measuring the emitted fluorescence instead to count each time the number of cells as usually performed with the traditional primary or transformed cell lines.

\section{Conclusions}

In conclusion our cellular model reduces 1) the cost of 
the experiments; 2) the time of the execution; 3) while giving the same kind of results obtained with other cellular models, as demonstrate herein in assaying the well characterized HA biomaterials $[9-11,14]$.

\section{Acknowledgements}

This study was supported in part by grants to M.T and P.F.N. from the Ministero della Università e Ricerca, University of Ferrara and University of Verona. Italy.

Dr. Katia Campioni was supported by a post-doc fellowship of the Fondazione Cassa di Risparmio di Cento, Italy.

SEM analyses were carried out at the Centre of Electron Microscopy, University of Ferrara, Ferrara, Italy.

\section{REFERENCES}

[1] J. A. Hubbell, "Bioactive Biomaterials," Current Opinion in Biotechnology, Vol. 10, No. 2, 1999, pp. 123-129.

[2] L. F. Reichardt, "Introduction: Extracellular Matrix Molecules. Guidebook to the Extracellular Matrix, Anchor, and Adhesion Proteins," Oxford University Press, New York, 1999 , p. 335.

[3] E. H. Danen and A. Sonnenberg, "Integrins in Regulation of Tissue Development and Function," The Journal of Pathology, Vol. 201, No. 4, 2003, pp. 632-641.

[4] M. M. Sandberg and H. T. Aro, "Gene Expression during Bone Repair," Clinical Orthopaedics and Related Research, Vol. 289, 1993, pp. 292-312.

[5] T. T. Kon and J. Cho, "Expression of Osteoprotegerin, Receptor Activator of NF-kappaB Ligand (Osteoprotegerin Ligand) and Related Proinflammatory Cytokines During Fracture Healing," Journal of Bone and Mineral Research, Vol. 16, No. 6, 2001, pp. 1004-1014.

[6] J. R. Lieberman and A. Daluiski, "The Role of Growth
Factors in the Repair of Bone. Biology and Clinical Applications." Journal of Bone Joint and Surgery, Vol. 84, No. A-6, 2002, pp. 1032-1044.

[7] D. M. Roy and S. K. Linnehan, "Hydroxyapatite Formed from Coral Skeletal Carbonate by Hydrothermal Exchange," Nature, Vol. 247, No. 438, 1974, pp. 220-222.

[8] A. R. Vaccaro, K. Chiba, et al., "Bone Grafting Alternatives in Spinal Surgery," The Spine Journal, Vol. 2, No. 3, 2002, pp. 206-215.

[9] O. Shamsuria, A. S. Fadilah, A. B. Asiah, M. R. Rodiah, A. H. Suzina and A. R. Samsudin "In Vitro Cytotoxicity Evaluation of Biomaterials on Human Osteoblast Cells CRL-1543; Hydroxyapatite, Natural Coral and Polyhydroxybutarate," Medical Journal of Malaysia, Vol. 59, Suppl. B, 2004, pp. 174-175.

[10] M. Barron, L. Franklin, J. Woodall Jr., S. Wingerter, H. Benghuzzi and M. Tucci, "Comparison of Osteoconductive Materials on MG63 Osteoblast Cell Function," Biomedical Sciences Instrumentation, Vol. 43, 2007, pp. 248-253.

[11] J. C. Fricain, J. Alouf, et al., "Cytocompatibility Study of Organic Matrix Extracted from Caribbean Coral Porites Astroides," Biomaterials, Vol. 23, No. 3, 2002, pp. 673-679.

[12] C. Morelli, G. Barbanti-Brodano, et al., "Cell Morphology, Markers, Spreading, and Proliferation on Orthopaedic Biomaterials. An Innovative Cellular Model for the In Vitro Study," The Journal of Biomedical Materials Research, Vol. 83, No. 1, 2007, pp. 178-183.

[13] M. Tognon, C. Morelli, et al., "A Novel Genetically Engineered Human Osteoblasts for the in Vitro Study of Biomaterials," In: N. Ashammakhi, R. Reis and F. Chiellini, Eds., Topics in Tissue Engineering, 2008, pp. 1-13.

[14] C.T. Begley, M. J. Doherty, D. P. Hankey and D. J. Wilson, "The Culture of Human Osteoblasts upon Bone Graft Substitutes," Bone, Vol. 14, No. 4, 1993, pp. 661-666. 